\title{
Cognitive behavioral therapy may have a rehabilitative, not normalizing, effect on functional
}

\section{connectivity in adolescent depression}

Villa $\mathrm{LM}^{1}$, Goodyer $\mathrm{IM}^{1}$, Tait $\mathrm{R}^{5}$, Kelvin $\mathrm{R}^{1,6,7}$, Reynolds $\mathrm{S}^{8,9}$ Wilkinson PO ${ }^{1,4}$, Suckling J 1,2,3,4

1: Department of Psychiatry, University of Cambridge

2: Behavioural and Clinical Neuroscience Institute, University of Cambridge

3: NIHR Cambridge Biomedical Research Centre

4: Cambridge and Peterborough NHS Foundation Trust

5: Faculty of Computing, Engineering and the Built Environment, Birmingham City University

6: The Royal College of Psychiatrists

7: The Association for Child and Adolescent Mental Health

8: School of Psychology and Clinical Language Sciences, University of Reading

9: Charlie Waller Institute, University of Reading

Corresponding Author: Luca Villa ${ }^{1}$

\footnotetext{
${ }^{1}$ Corresponding author: Luca Villa

Mailing address: Herchel Smith Building, Robinson Way, Cambridge, CB2 OSZ

Email: Imv31@cam.ac.uk
}

Phone Number: 07921827103 


\section{Abstract}

Background: Whether the differences in brain structure and function, characteristic of adult major depressive disorder $\left(\mathrm{MDD}^{2}\right)$, are present in adolescent $\mathrm{MDD}$ is still unclear, but it has been shown that cognitive behavioral therapy $\left(\mathrm{CBT}^{3}\right)$ affects resting-state functional connectivity in both adult and adolescent MDD patients, with the claim that CBT has a normalizing effect on MDD-related functional disruption, but this has not been directly tested.

Methods: 128 adolescent MDD patients and 40 adolescent controls were enrolled in the study. We investigated pre-treatment differences in cortical thickness, white matter volume, and resting-state functional connectivity. We also investigated the longitudinal effects of $C B T$ on resting-state functional connectivity, and the relationship between pre-treatment functional disruption and CBTrelated changes to resting-state functional connectivity was assessed by the correlation of pretreatment cross-sectional effects and longitudinal CBT-related effects across multiple brain regions.

Results: Patients had greater cortical thickness and white matter volume within fronto-limbic regions of the brain. Patients had greater pre-treatment resting-state functional connectivity within the default-mode, fronto-limbic, central-executive, and salience networks. CBT increased restingstate functional connectivity of the subgenual anterior cingulate and amygdala seeds with predominantly frontal regions. Regions showing the greatest pre-treatment functional disruption showed the weakest CBT-related changes.

Limitations: For ethical reasons, there was no placebo group.

Conclusions: Adolescent MDD is associated with structural and functional differences also seen in adult patients. CBT-related changes in resting-state functional connectivity do not appear to show a normalizing effect, but instead indicate rehabilitative effects on resting-state functional connectivity.

\footnotetext{
${ }^{2}$ Major Depressive Disorder (MDD)

${ }^{3}$ Cognitive Behavioural Therapy (CBT)
} 
Keywords: Adolescent depression, MRI, CBT, Cortical thickness, Resting-state functional connectivity, White matter volume 


\section{Introduction}

Although cognitive behavioral therapy (CBT) is a clinically effective treatment for both adult and adolescent major depressive disorder (MDD; DeRubeis et al., 2008; Weersing et al., 2017) its effects within the brain are still poorly understood. Uncovering the neurobiological characteristics of MDD, both before and after receiving CBT, may be vital to understanding the processes unfolding within the depressed brain.

Whilst there is a considerable volume of literature exploring case-control differences in brain structure and function in adult MDD (Bora et al., 2012; Kaiser et al., 2015; Mulders et al., 2015), it is still unclear to what extent the adolescent brain is affected by the illness. There have been inconsistencies between adult and adolescent MDD patients regarding their brain structure. Studies using adult MDD patients often find deviations in cortical thickness (Grieve et al., 2013; Schmaal et al., 2017), grey matter volume (Bora et al., 2012; Grieve et al., 2013), and white matter volume (Zeng et al., 2012), whereas those using adolescent MDD patients are often unable to find any structural deviations (Hagan et al., 2015; Schmaal et al., 2017) or instead find the opposite effects to those of adult MDD samples (Steingard et al., 2002). These inconsistencies in brain structure between adult and adolescent MDD patients remain unexplained, making it unclear whether structural deviations are actually present in adolescent MDD.

As well as brain structure, adult MDD patients have shown differences in brain function, particularly in resting-state functional connectivity ( $\mathrm{rFF}$ ). Key functional networks implicated in adult MDD are the fronto-limbic network, involved in emotional reactivity and regulation (Casey et al., 2010; Mayberg, 1997; Roiser and Sahakian, 2013; Wang et al., 2012), the default-mode network, involved 
in internal thoughts and self-referential processing (Kaiser et al., 2015; Mulders et al., 2015), the central-executive network, involved in tasks that require executive function (Kaiser et al., 2015; Mulders et al., 2015), and the salience network, involved in switching between the default-mode and central-executive networks, and in directing attention towards emotionally salient stimuli (Manoliu et al., 2014; Mulders et al., 2015; Ramasubbu et al., 2014), see supplementary information (S1). Again, when studying adolescent MDD, the literature is less clear with only some studies replicating previous findings in adult MDD patients (Connolly et al., 2013; Ho et al., 2015), others reporting opposite effects (Mulders et al., 2015), or being unable to obtain replication (Pannekoek et al., 2014).

The lack of coherence within the adolescent MDD literature highlights a need to further investigate brain structure and rsFC in adolescent MDD. Furthermore, a link between structural and functional deviations in MDD has previously been established in adult patients, with it being suggested that changes in brain structure may cause the functional disruption seen in adult MDD (De Kwaasteniet et al., 2013; Ma et al., 2012; Späti et al., 2015; Van Tol et al., 2014), and yet the same has not been done in adolescent MDD. If structural and functional deviations, similar to those found in adult MDD, do occur in adolescent MDD, understanding their relationship could be vital in uncovering the nature of the illness in adolescence.

From a therapeutic perspective, there is some evidence that psychological treatment exerts effects that may resolve neural differences associated with current MDD, which does appear to occur in both adult and adolescent MDD patients. CBT has been found to affect rsFC within the fronto-limbic network, in adult MDD, thereby possibly normalizing functional disruption within frontal regions of the brain (Goldapple et al., 2004; Ritchey et al., 2011; Shou et al., 2017). Interestingly, the sensitivity of rsFC to CBT appears to translate to adolescent MDD patients; for example, it has been found that 
receiving rumination focused-CBT may decrease rsFC within the default-mode network in adolescent MDD patients (Jacobs et al., 2016). This may suggest that treatment normalizes disruption in rsFC whilst depressed (Jacobs et al., 2016; Ritchey et al., 2011; Shou et al., 2017), regardless of age at presentation. However, the relationship between pre-treatment functional disruption and later treatment effects in adolescent MDD has not been explored. Therefore, although it is thought that CBT normalizes MDD-related functional disruption, this has not been directly tested by investigating whether regions showing the greatest functional disruption actually show the greatest CBT-related changes in rsFC, highlighting a fundamental gap in our understanding.

The present study investigated brain structure and function in adolescent MDD, and their relationship with each other. We examined the cortical thickness of the subgenual anterior cingulate and medial orbitofrontal cortex as they have previously been implicated with adult MDD (Grieve et al., 2013; Hagan et al., 2013; Peterson et al., 2009; Schmaal et al., 2017). Additionally, we investigated the white matter volume of the medial frontal gyrus, middle frontal gyrus, superior frontal gyrus, and medial orbitofrontal cortex, as frontal white matter volume has been implicated in both adult and adolescent MDD (Steingard et al., 2002; Zeng et al., 2012). We expected to find a group-by-age interaction in both cortical thickness and white matter volume within the anterior cingulate cortex, as had previously been found in grey matter volume using our sample (Hagan et al., 2015). We further hypothesized that patients would show pre-treatment functional disruption in regions within the fronto-limbic, default-mode, central-executive, and salience networks, which have been implicated in studies of adult MDD (Kaiser et al., 2015; Mulders et al., 2015). However, due to the inconsistency within the literature, we did not hypothesize the direction of this disruption. We did hypothesize that any deviations in brain structure and function would be correlated with each other. 
Additionally, we investigated the longitudinal effects of $\mathrm{CBT}$ on $\mathrm{rSFC}$, in patients, and in particular the relationship between pre-treatment functional disruption and later CBT-related changes in rsFC, hypothesizing that if CBT has a normalizing effect on brain function, as has previously been suggested (Jacobs et al., 2016; Ritchey et al., 2011; Shou et al., 2017; Yoshimura et al., 2017), then regions of the brain showing the greatest pre-treatment functional disruption would also receive the greatest impact from CBT. 


\section{Methods}

Study overview

The IMPACT study (Goodyer et al., 2017) was a randomized controlled superiority trial assessing medium-term effects of psychological therapies in adolescent MDD. Patients $(n=470)$ met diagnostic requirements for MDD using the DSM-IV (American Psychiatric Association, 1994) and were randomized to treatment arms of either CBT, short-term psychoanalytical therapy, or a brief psychological intervention, and were followed-up over an 86 week period.

A subset of patients enrolled in IMPACT were recruited for the MR-IMPACT study ( $n=128$; Hagan et al., 2015). These patients were assessed using structural and functional MRI at an initial baseline scan, prior to starting treatment. The Short Moods and Feelings Questionnaire (SMFQ; Sharp et al., 2006) and State-Trait Anxiety Inventory ( STAl; Spielberger et al., 1970) were administered on the day of scanning as a measure of immediate depression and anxiety symptoms. Those randomized to the CBT arm of IMPACT $(n=20)$ were invited to attend a second, six-month post-treatment assessment, with the requirement of having received at least six sessions of therapy. Additionally, adolescent controls ( $n=40$ ), with no history of MDD and no first-degree relatives with MDD, were recruited for MR-IMPACT (Hagan et al., 2015, 2013) and attended two assessments, separated by six months. The study received favorable ethical opinion by the National Research Ethics Service Committee East of England (reference: 09/H0308/168).

Previous work focusing on task-based fMRI, using this sample, are reported in Chuang et al. (2016), Chuang et al. (2017) and Holt et al. (2016). 


\section{Participant samples}

There were no significant differences $(p>0.05)$ between controls and patients on age and gender, shown in supplementary information (S2).

In total, 128 patients and 40 controls attended the baseline scan. For structural scans, the data from 19 patients and 4 controls were excluded, detailed in supplementary information (S3). This left a sample of 109 patients and 36 controls who had structural data - see Table 1 for participant demographics. No structural MRI data were collected post-treatment.

For functional scans, patients with MDD were separated into two independent samples: a functional cross-sectional sample of patients who only attended the baseline scan, and a functional longitudinal sample of patients who attended both the baseline scan and the later post-treatment follow-up scan. This separation was done to allow independence of the two patient groups when comparing any initial case-control differences to CBT-related changes in rSFC. The same data from control participants were used with both cross-sectional and longitudinal samples, due to small sample sizes.

There were 108 patients and 40 controls within the cross-sectional sample, and 20 patients and 33 controls within the longitudinal sample. Of these, the data from 29 patients and 9 controls were excluded - shown in supplementary information (S3). This left functional data for 82 patients and 34 controls within the functional cross-sectional sample, and for 17 patients and 30 controls within the functional longitudinal sample. 
Seventy-five patients within the functional cross-sectional sample had both structural and functional data that were used to investigate the relationship between brain structure and function in patients.

Table 1. Showing participant characteristics with standard deviations (SD) in brackets.

\begin{tabular}{|c|c|c|c|c|c|c|c|c|c|c|}
\hline & $\begin{array}{l}\text { First } \\
\text { Mean } \\
\text { Age } \\
\text { (SD), } \\
\text { range }\end{array}$ & $\begin{array}{c}\text { First } \\
\text { Gender } \\
\text { (\% Female) }\end{array}$ & $\begin{array}{c}\text { First } \\
\text { Mean } \\
\text { SMFQ } \\
\text { Score }\end{array}$ & $\begin{array}{c}\text { First } \\
\text { Mean } \\
\text { STAIT } \\
\text { Score }\end{array}$ & $\begin{array}{l}\text { First } \\
\text { Mean } \\
\text { STAIS } \\
\text { Score }\end{array}$ & $\begin{array}{c}\text { Second } \\
\text { Mean Age } \\
\text { (SD), range }\end{array}$ & $\begin{array}{c}\text { Second } \\
\text { Gender } \\
\text { (\% Female) }\end{array}$ & $\begin{array}{c}\text { Second } \\
\text { Mean } \\
\text { SMFQ } \\
\text { Score }\end{array}$ & $\begin{array}{c}\text { Second } \\
\text { Mean } \\
\text { STAIT } \\
\text { Score }\end{array}$ & $\begin{array}{c}\text { Second } \\
\text { Mean } \\
\text { STAIS } \\
\text { Score }\end{array}$ \\
\hline \multicolumn{11}{|l|}{ Structure } \\
\hline $\begin{array}{l}\text { Structural } \\
\text { Patients, } \\
n=109\end{array}$ & $\begin{array}{c}15.56 \\
(1.27), \\
11.83- \\
17.96\end{array}$ & 74.31 & $\begin{array}{l}19.20 \\
(6.95)\end{array}$ & $\begin{array}{l}60.12 \\
(7.89)\end{array}$ & $\begin{array}{c}45.62 \\
(10.91)\end{array}$ & $\mathrm{N} / \mathrm{A}$ & N/A & $\mathrm{N} / \mathrm{A}$ & N/A & N/A \\
\hline $\begin{array}{l}\text { Structural } \\
\text { Controls, } \\
n=36\end{array}$ & $\begin{array}{c}15.65 \\
(1.45), \\
12.14- \\
17.73\end{array}$ & 72.22 & $\begin{array}{c}3.03 \\
(1.93)\end{array}$ & $\begin{array}{l}31.03 \\
(6.39)\end{array}$ & $\begin{array}{l}29.56 \\
(6.43)\end{array}$ & $\mathrm{N} / \mathrm{A}$ & N/A & N/A & $\mathrm{N} / \mathrm{A}$ & N/A \\
\hline \multicolumn{11}{|l|}{ Function } \\
\hline $\begin{array}{l}\text { Cross-Section } \\
\text { Patients, } \\
n=82\end{array}$ & $\begin{array}{c}15.69 \\
(1.12), \\
13.48- \\
17.96 \\
\end{array}$ & 78.05 & $\begin{array}{l}19.98 \\
(7.18)\end{array}$ & $\begin{array}{l}59.78 \\
(8.02)\end{array}$ & $\begin{array}{c}45.93 \\
(10.01)\end{array}$ & N/A & $\mathrm{N} / \mathrm{A}$ & N/A & $\mathrm{N} / \mathrm{A}$ & N/A \\
\hline $\begin{array}{l}\text { Cross-Section } \\
\text { Controls, } \\
n=34\end{array}$ & $\begin{array}{c}15.73 \\
(1.44), \\
12.14- \\
17.73\end{array}$ & 79.41 & $\begin{array}{c}2.71 \\
(1.99)\end{array}$ & $\begin{array}{l}30.59 \\
(6.58)\end{array}$ & $\begin{array}{l}28.85 \\
(6.75)\end{array}$ & $\mathrm{N} / \mathrm{A}$ & N/A & $\mathrm{N} / \mathrm{A}$ & N/A & N/A \\
\hline $\begin{array}{l}\text { Longitudinal } \\
\text { Patients, } \\
n=17\end{array}$ & $\begin{array}{c}15.42 \\
(1.37), \\
12.89- \\
17.56\end{array}$ & 82.35 & $\begin{array}{l}18.12 \\
(6.48)\end{array}$ & $\begin{array}{l}60.35 \\
(8.02)\end{array}$ & $\begin{array}{c}48.24 \\
(11.33)\end{array}$ & $\begin{array}{c}16.07(1.34) \\
13.41-18.17\end{array}$ & 82.35 & $\begin{array}{c}9.00 \\
(5.28)\end{array}$ & $\begin{array}{c}44.17 \\
(10.48)\end{array}$ & $\begin{array}{c}35.71 \\
(10.14)\end{array}$ \\
\hline $\begin{array}{l}\text { Longitudinal } \\
\text { Controls, } \\
n=30\end{array}$ & $\begin{array}{c}15.59 \\
(1.47), \\
12.14- \\
17.73\end{array}$ & 80.00 & $\begin{array}{c}2.60 \\
(1.77)\end{array}$ & $\begin{array}{l}30.50 \\
(6.91)\end{array}$ & $\begin{array}{l}28.93 \\
(6.89)\end{array}$ & $\begin{array}{c}16.24(1.46) \\
12.92-18.30\end{array}$ & 80.00 & $\begin{array}{c}2.21 \\
(1.81)\end{array}$ & $\begin{array}{l}28.68 \\
(6.62)\end{array}$ & $\begin{array}{l}26.43 \\
(6.30)\end{array}$ \\
\hline
\end{tabular}


Analyses for structural case-control differences

MRI acquisition and pre-processing details are shown in supplementary information (S4).

A mask covering the subgenual anterior cingulate and medial orbitofrontal cortex was used for the investigation of case-control differences in cortical thickness. These regions were chosen based on previous findings in the literature that had associated them with MDD (Grieve et al., 2013; Peterson et al., 2009; Schmaal et al., 2017) and pre-specified as regions of interest in the study protocol (Hagan et al., 2013). The Automated Anatomical Labelling (AAL) atlas (Tzourio-Mazoyer et al., 2002) was used to identify the medial orbitofrontal cortex. To identify the subgenual anterior cingulate, the anterior cingulate cortex regions of the AAL template were divided into the pregenual anterior cingulate and subgenual anterior cingulate - shown in supplementary information (S5).

For white matter volume analyses, a mask encompassing the medial frontal gyrus, middle frontal gyrus, superior frontal gyrus, and medial orbitofrontal cortex was used, based on previous findings within the literature (Steingard et al., 2002; Zeng et al., 2012). These regions were identified using the AAL atlas (Tzourio-Mazoyer et al., 2002).

An ANCOVA was used to investigate case-control differences at each voxel within the mask, with group (patient or control) as the independent variable and age (years) and gender values as covariates. For inference of the results of the general linear model, permutation-based methods were used with FSL Randomise (Jenkinson et al., 2012). This procedure conducted 100,000 permutations per statistical test, using threshold-free cluster enhancement, with a Family-wise error $(p<0.05)$ correction to account for multiple comparisons, generating clusters of significant casecontrol differences. Cohen's $d$ (Cohen, 1988) was used as a measure of effect size. We also investigated cortical surface area, detailed in supplementary information (S6) 
Analyses for structural group-by-age interactions

A mask of the entire anterior cingulate cortex was used to investigate group-by-age interactions in both cortical thickness and white matter volume. The AAL template (Tzourio-Mazoyer et al., 2002) was used to identify the anterior cingulate cortex.

An ANCOVA was applied to investigate group-by-age interactions, with gender as a covariate, using the same permutation-based inference as previously described.

\section{Analyses for functional case-control differences}

Seed-based methods were used to investigate rsFC of functional brain networks, with seeds located bilaterally. For the fronto-limbic network, the medial orbitofrontal cortex, hippocampi, and medial frontal gyri were used as seed regions. For the default-mode network, the precuneus and the subgenual anterior cingulate were used. For the central-executive network, the middle frontal gyri and superior frontal gyri were seed regions. For the salience network, the amygdalae were used. The boundaries of the seed regions were defined from the AAL atlas, except for the subgenual anterior cingulate seeds, which were identified using the same methods previously described for structural analyses.

For each seed, an ANCOVA was used to investigate case-control differences at each voxel across a mask, excluding voxels within the seed region. As multiple seed regions, such as the hippocampus and subgenual anterior cingulate, are involved in multiple brain networks, the mask covered regions of all four networks. Definitions of which regions were encompassed within this mask are shown in 
supplementary information (S7). Age and gender were included as covariates. This model was regressed on the baseline data of the cross-sectional sample. Statistical inferences were again conducted using the same permutation methods previously described.

\section{Analyses for CBT-related changes in resting-state functional connectivity}

Seed regions that showed pre-treatment differences in rsFC, within the cross-sectional sample, were chosen as seed regions to investigate CBT-related changes in rSFC within the longitudinal sample.

Seed-based rsFC maps from each participant's post-treatment functional scan were subtracted from their respective pre-treatment rsFC maps and then between-group comparisons were made, controlling for age changes and gender, using the same permutation methods as previously described. This is equivalent to testing for a group-by-time interaction in a $2 \times 2$ design.

Although there was no placebo group in this study, due to the ethical issues of restricting treatment to some patients, we refer to group-by-time interactions as CBT-related changes.

\section{Post-Hoc Correlations}

SMFQ and STAI scores were correlated with any significant regions of pre-treatment structural and functional differences in patients. Significant structural and functional clusters within the crosssectional sample were correlated with each other for the 75 cross-sectional patients who had both structural and functional data.

Significant functional clusters showing CBT-related changes in patients within the longitudinal sample were correlated with changes in symptoms. The change in rsFC, relative to baseline rsFC, within the largest significant cluster of each seed was correlated with the slope of symptom changes 
in SMFQ and STAI scores. All correlations were corrected using the false discovery rate (Hochberg, 1995). Further details are given in supplementary information (S8).

Relationship between pre-treatment functional disruption and later CBT-related changes

Each case-control and group-by-time interaction seed analysis produced a $z$-score map for their respective effects. In other words, the case-control z-score maps show regions of MDD-related pretreatment functional disruption within the cross-sectional sample. Similarly, group-by-time interaction z-score maps show regions exhibiting CBT-related changes within the longitudinal sample. We aimed to investigate whether regions showing the greatest pre-treatment functional disruption would also show the greatest CBT-related changes by investigating the relationship between case-control z-scores and group-by-time interaction $z$-scores.

For this approach, we parcellated the brain into 118 regions; each region was a parcellation of the AAL template, except for the anterior cingulate cortex which was sub-divided into the pregenual anterior cingulate and subgenual anterior cingulate using the previously described ROIs. We conducted two sets of 118 seed-based rsFC analyses, one set of the case-control analyses - using the cross-sectional sample - and another set of the group-by-time analyses - using the longitudinal sample.

Each seed analysis produced a voxel-wise z-score map across the brain for case-control and groupby-time effects from which 117 mean $z$-scores were obtained (i.e. number of parcels minus the seed 
region). For each seed and each corresponding parcel ("target region"), correlations were calculated between the case-control mean $z$-scores with the corresponding mean group-by-time $z$-scores across the voxels of the region. The procedure is illustrated in Figure 1.

As almost all of these correlations involved thousands of individual voxels, we did not conduct significance testing as very small effects are significant even at small $p$-value thresholds. Instead, correlation coefficients were converted into $r^{2}$ as a measure of effect size.

To investigate the relationship between pre-treatment functional disruption and CBT-related changes at the network level, seed regions were grouped into the default-mode, fronto-limbic, central-executive, and salience networks, and the effect sizes of their target regions were averaged across their network. Effect sizes of positive and negative correlations were treated separately when averaging. We also averaged the target region effect sizes across all 118 seed regions to identify the relationship between pre-treatment functional disruption and CBT-related changes across the whole brain. 


\section{Results}

For brevity, exploratory whole-brain structural analyses and non-significant results are shown in supplementary information (S9).

\section{$\underline{\text { Brain Structure }}$}

\section{Cortical Thickness}

Contradicting our hypothesis that there would be no structural differences, the bilateral subgenual anterior cingulate and medial orbitofrontal cortex showed greater cortical thickness in patients, $t(143)=3.57854$, pcorr $=0.022, d=0.771 ;$ Figure 2.

There were no significant relationships between mean cortical thickness in the cluster and current symptoms of depression and anxiety.

In agreement with our hypothesis, we found a group-by-age interaction, in cortical thickness, within the left and right pregenual anterior cingulate, $t(143)=3.55955$, pcorr $=0.014$; Figure 2 .

\section{White Matter Volume}

Contradicting our hypothesis that there would be no structural differences, patients showed greater white matter volume within the frontal regions of the brain, $t(143)=3.53034$, pcorr $=0.017, d=$ 0.708 , specifically in the bilateral medial frontal gyri, bilateral superior frontal gyri and right middle frontal gyrus; Figure 2. There was no significant correlation between mean white matter volume and mean cortical thickness in their respective clusters, $r=-0.017, p$ corr $=0.861$. 
There were no significant relationships between mean white matter volume in the cluster and current symptoms of depression and anxiety.

In agreement with our hypothesis, we found a group-by-age interaction, in white matter volume, within the bilateral pregenual anterior cingulate and the right subgenual anterior cingulate, $t(143)=$ 3.17165, pcorr = 0.022; Figure 2 .

\section{$\underline{\text { Brain Function }}$}

Only the largest clusters of each seed region are shown. Smaller functional clusters are detailed in supplementary information (S10).

Resting State Functional Connectivity of the Cross-sectional Sample

In agreement with our hypothesis that there would be pre-treatment case-control differences, various regions showed greater rsFC with the right superior frontal gyrus, right subgenual anterior cingulate, and right amygdala seeds, in patients; Figure 4 and S10 in supplementary information. There were no significant correlations between mean regional rsFC and current symptoms of depression and anxiety.

Replication of these case-control analyses using the longitudinal sample are detailed in supplementary information (S11). 
Associated with the left amygdala and the left subgenual anterior cingulate seeds, a variety of regions showed significant group-by-time interactions in rsFC; Figure 4 and S12 in supplementary information. In all significant interactions, rsFC with the seed region had increased in patients after treatment.

There was no relationship between any of the CBT-related changes and changes in symptoms.

Relationship between structural and functional differences

There were no significant correlations between any significant functional clusters and structural clusters.

Relationship between pre-treatment functional disruption and CBT-related changes

Strikingly, although we hypothesized that regions showing the greatest pre-treatment functional disruption would show the greatest CBT-related changes in rsFC, producing positive correlations between case-control and group-by-time interaction z-scores, few correlations were actually positive. Of the 13,806 correlations only 2025 (14.67\%) were positive; supplementary information (S13).

The $r^{2}$ values of the positive correlations, between pre-treatment case-control $z$-scores and groupby-time interaction $z$-scores, ranged between $5.979 \times 10^{-9}-0.8177$, although almost all were weak, with the median $r^{2}=0.0082$. Only $5 \%$ of the positive correlations had $r^{2}>0.15$; supplementary 
information (S13). The $r^{2}$ values of positive correlations, averaged across functional networks and the whole brain are shown in Figure 5.

The $r^{2}$ values of the negative correlations, between pre-treatment case-control $z$-scores and groupby-time interaction $z$-scores, ranged between $8.3244 \times 10^{-10}-0.8062$, with a median $r^{2}$ of 0.0716 . The distribution of these negative correlations is shown in supplementary information (S13), with $25 \%$ of the correlations having an $r^{2}>0.15$. The $r^{2}$ values of negative correlations, averaged across functional networks and the whole brain are shown in Figure 5.

The top $10 \%$ of target regions, showing the strongest averaged effect sizes for negative correlations, between pre-treatment case-control z-scores and group-by-time interaction z-scores, across the seed regions grouped into networks and the whole brain, are shown in Figure 5. Rankings of all target regions, showing the strongest averaged effect sizes for negative correlations, across the seed regions grouped into networks, are shown in supplementary information (S14). 


\section{Discussion}

Adolescent MDD patients had greater cortical thickness within the subgenual anterior cingulate and medial orbitofrontal cortex, and greater white matter volume within frontal regions as well as group-by-age interactions within the anterior cingulate cortex in both cortical thickness and white matter volume. Patients also showed greater rsFC with the right superior frontal gyrus, amygdala and subgenual anterior cingulate seeds, and CBT-related changes (group-by-time interactions) between predominantly frontal regions and the left subgenual anterior cingulate and amygdala seeds, with rsFC increasing following CBT. Regions showing the greatest functional disruption often later showed the weakest $\mathrm{CBT}$-related changes in $\mathrm{rSFC}$, with this effect being most prominent within the medial orbitofrontal cortex.

MDD-related reductions in cortical thickness, within the subgenual anterior cingulate and medial orbitofrontal cortex, have previously been reported in adult MDD patients (Peterson et al., 2009; Schmaal et al., 2017), which is contrary to our adolescent patients where cortical thickness was greater. However, although the literature is inconsistent, cortical thickening in adolescent MDD has previously been found and explained as being caused by increased neuronal numbers or neural density (Reynolds et al., 2014). Moreover, several longitudinal studies have linked cortical thickness reductions during brain maturation to myelination across the grey-white matter boundary (Sowell et al., 2003; Whitaker et al., 2016). Therefore, greater cortical thickness within the subgenual anterior cingulate and medial orbitofrontal cortex found in patients may be due to either a delay or premature curtailment of this neurodevelopmental process. The greater white matter volume within the frontal lobes of patients does suggest that deviating white matter developmental trajectories are taking place, further evidenced by the group-by-age interactions, between patients and controls, found in the anterior cingulate cortex both in cortical thickness and white matter volume. From a 
neurobiological view, spatially localized increases in both cortical thickness and white matter volume appear to be contradictory, although a single process may underlie both observations, with myelination across the grey-white matter boundary being hindered or delayed in adolescent MDD and instead increasing within the white matter tract.

The largest study investigating cortical thickness in adolescent MDD, comparing 213 adolescent MDD patients to 294 controls, found no group differences in cortical thickness, instead finding differences in cortical surface area (Schmaal et al., 2017). Conversely, we found that adolescent MDD patients showed both case-control differences and a group-by-age interaction in cortical thickness, but no differences in cortical surface area (supplementary information, S6). The reason for this discrepancy may be due to the substantial age differences between these two patient samples. In Schmaal et al.'s (2017) study, $70 \%$ of their adolescent sample were between the ages of $18-21$ years, with only 64 of their adolescent MDD patients below 18 years. Contrastingly, with a mean age of 15.56 years, none of our 109 MDD patients used for the structural analyses were above 18 years, and thus were substantially younger than those in Schmaal et al. (2017). Furthermore, and as conceded by Schmaal et al. (2017), early adolescence may be a more sensitive period for structural deviations to occur in adolescent MDD, and their use of a substantially older adolescent sample may have hindered their ability to detect deviations in cortical thickness.

Patients in the cross-sectional sample showed greater rsFC across the fronto-limbic, default-mode, central-executive and salience networks, demonstrating disruption to similar networks that have been implicated in the adult MDD literature (Casey et al., 2010; Kaiser et al., 2015; Manoliu et al., 2014; Mayberg, 1997; Mulders et al., 2015; Ramasubbu et al., 2014; Roiser and Sahakian, 2013; Wang et al., 2012). However, the degree of the similarity regarding the direction of this functional disruption is still unclear due to inconsistencies across these studies. Strikingly, none of these 
significant functional differences were correlated with symptoms of depression and anxiety. It may be that the measures of general depression and anxiety that were used are not sensitive enough or are too broad to be related to the pre-treatment functional disruption that we found. Moreover, it could also be that the observed pre-treatment functional disruption is instead a trait marker of adolescent MDD, rather than a marker of current depressed state.

The spatial convergence between seeds showing pre-treatment functional disruption and regions showing structural deviations in cortical thickness and white matter volume, being the subgenual anterior cingulate and superior frontal gyrus, hints towards an underlying process that might be causing both structural and functional deviations within the same regions. However, the lack of correlation between the seed-based functional clusters and structural clusters contradicts this possibility and is striking given that the past literature has found strong relationships between structural deviation and functional disruption in adult MDD (De Kwaasteniet et al., 2013; Ma et al., 2012; Späti et al., 2015; Van Tol et al., 2014). As the pre-treatment functional clusters were large and spanned across multiple regions of the brain, simply correlating clusters of deviating cortical thickness and white matter volume with such large functional clusters may have been too imprecise to find a significant relationship. Conversely, it may instead be that in adolescent MDD, although some brain regions show both structural and functional deviations, they have independent causes and do not interact with each other. Longitudinal tracking of structural deviations in adolescent MDD and how they relate to longitudinal changes in rSFC may help explain this issue.

A key focus of this study was the relationship between the magnitude and location of pre-treatment functional disruption and CBT-related changes in rsFC. The vast majority of the correlations between pre-treatment case-control $z$-scores and group-by-time interaction $z$-scores were negative with this effect often being strongest within the medial orbitofrontal cortex as, when examining the four 
major functional networks and the whole brain, it consistently showed some of the strongest negative correlations. These results suggest that CBT-related changes in rsFC are weaker within regions that show the greatest pre-treatment functional disruption and do not show normalizing effects, as if that were the case CBT-related changes in rsFC would be greatest in regions showing the greatest functional disruption.

Why regions with strongly affected $\mathrm{rsFC}$ might be less amenable to $\mathrm{CBT}$ is uncertain. Regions showing the greatest functional disruption may simply take more time to demonstrate CBT-related changes, and a six-month interval, although accompanied by large symptom improvements, was insufficient to see the full effects of CBT. Alternatively, regions showing the greatest functional disruption may become functionally impaired and less responsive to CBT. The maintenance of this effect by long-term observation of rsFC would resolve these possibilities.

Another possibility is that CBT functionally enhances brain regions that are unaffected by the illness, instead of normalizing affected regions. CBT aims to help patients achieve remission by modifying cognitive and behavioral aspects of adolescent MDD. This is done by increasing exposure to pleasurable behaviors, building skills, and by identifying cognitive biases, such as attentional and interpretation bias, and changing them to become less negative. This development of new skills, routines, and changes to thought processes may lead to functional compensation within the brain. This could explain why the observed CBT-related changes led to increased rsFC between the left subgenual anterior cingulate seed and frontal regions, rather than leading to normalizing effects, and more generally that regions of pre-treatment functional disruption did not overlap with the regions showing CBT-related changes. In future it may be more appropriate to focus on the relationship between treatment-related functional changes and potential CBT-related 
enhancements in specific cognitive or emotional domains, such as emotional regulation, rather than focusing solely on their relationship to a reduction in symptoms of depression.

A disruptive trend in rSFC as MDD progresses cannot be discounted. There was no placebo group within the study, meaning that the observed group-by-time interactions in rsFC may not actually demonstrate the direct effects of CBT. Regions of significant group-by-time interactions may in fact reflect patients' rsFC becoming further disrupted, rather than demonstrating CBT-related changes, which may account for the lack of correlations between changes in rsFC and changes in symptoms. Negative correlations between pre-treatment functional disruption and later CBT-related changes in rsFC may be due to a ceiling or non-linear effect in functional disruption, with some functionally disrupted regions being at maximum and incapable of further alteration. Such regions would then show the weakest changes over time compared to initially less affected regions with more scope for disruption. However, there is little evidence to support the existence of this ceiling effect, and the absence of a correlation between changes in rsFC and changes in symptoms may simply be due to the small sample size in the longitudinal sample.

In conclusion, adolescent MDD patients had greater cortical thickness and white matter volume and showed group-by-age interactions within the anterior cingulate cortex, which we consider point to putative deviating developmental neural trajectories rather than direct illness effects on brain regions. Functional disruption to the fronto-limbic, default-mode, central-executive, and salience networks, previously reported in adult MDD, appears to be present in adolescent MDD. Importantly, the relationship between pre-treatment functional disruption and CBT-related changes are mostly negative, with brain regions having the greatest pre-treatment functional disruption showing the weakest CBT-related changes in rsFC. With the rehabilitative nature of $\mathrm{CBT}$ in mind, this finding 
suggests that there is more to the neurobiological effects of CBT on adolescent MDD than a simple transition from functional disruption to normalization.

\section{Limitations}

The main limitation of this study is that there was no placebo group of MDD patients who were longitudinally assessed, due to the ethical issue of delaying treatment. The comparison group were healthy controls assessed longitudinally over the same period. The absence of a placebo group means that any putative CBT-related changes in rsFC in patients cannot be uniquely ascribed to receiving treatment and may instead be interpreted as being due to aging or maturational processes that are specific to adolescent MDD, or be part of the progression of MDD-related functional disruption.

As with all studies, a larger sample size and balancing of the control and patient groups would improve the statistical power to find case-control differences within the cross-sectional sample. Similarly, a small sample size in the longitudinal sample means our patient sample is potentially unrepresentative of the general adolescent MDD population and the results more susceptible to statistical outliers. Our sample is also likely to be affected by attritional bias, with those patients whose symptoms did not improve being more likely to withdraw from the study. 


\section{Acknowledgements}

The study was funded by the UK Medical Research Council (grant: G0802226), the National Institute for Health Research (NIHR) (grant: 06-05-01), financial support from the Department of Health, and the Behavioral and Clinical Neuroscience Institute (BCNI), University of Cambridge, the latter being jointly funded by the Medical Research Council and the Wellcome Trust. Additional support was received from the Cambridge Biomedical Research Centre.

We are extremely grateful to all participants for their contribution to this work. We also thank the role of the Wolfson Brain Imaging Centre, Cambridgeshire and Peterborough NHS Foundation Trust, Child and Adolescent Mental Health Services, Mental Health Research Network, IMPACT research assistants, IMPACT clinicians, and the IMPACT Consortium, without whom this study could not have taken place. Luca Villa is supported by the Oon Khye Beng Ch'hia Tsio Studentship from Downing College, University of Cambridge. 


\section{References}

American Psychiatric Association, 1994. Diagnostic and statistical manual of mental disorders (4th ed.), Diagnostic and statistical manual of mental disorders (4th ed.).

Bora, E., Fornito, A., Pantelis, C., Yücel, M., 2012. Gray matter abnormalities in Major Depressive Disorder: A meta-analysis of voxel based morphometry studies. J. Affect. Disord. https://doi.org/10.1016/j.jad.2011.03.049

Casey, B.J., Jones, R.M., Levita, L., Libby, V., Pattwell, S.S., Ruberry, E.J., Soliman, F., Somerville, L.H., 2010. The storm and stress of adolescence: Insights from human imaging and mouse genetics. Dev. Psychobiol. https://doi.org/10.1002/dev.20447

Chuang, J.Y., Hagan, C.C., Murray, G.K., Graham, J.M.E., Ooi, C., Tait, R., Holt, R.J., Elliott, R., van Nieuwenhuizen, A.O., Bullmore, E.T., Lennox, B.R., Sahakian, B.J., Goodyer, I.M., Suckling, J., 2017. Adolescent major depressive disorder: Neuroimaging evidence of sex difference during an affective Go/No-Go task. Front. Psychiatry. https://doi.org/10.3389/fpsyt.2017.00119

Chuang, J.Y., Whitaker, K.J., Murray, G.K., Elliott, R., Hagan, C.C., Graham, J.M.E., Ooi, C., Tait, R., Holt, R.J., Van Nieuwenhuizen, A.O., Reynolds, S., Wilkinson, P.O., Bullmore, E.T., Lennox, B.R., Sahakian, B.J., Goodyer, I., Suckling, J., 2016. Aberrant brain responses to emotionally valent words is normalised after cognitive behavioural therapy in female depressed adolescents. J. Affect. Disord. https://doi.org/10.1016/j.jad.2015.09.008

Cohen, J., 1988. Statistical power analysis for the behavioral sciences, second edition, Statistical Power Analysis for the Behavioral Sciences. https://doi.org/10.1234/12345678

Connolly, C.G., Wu, J., Ho, T.C., Hoeft, F., Wolkowitz, O., Eisendrath, S., Frank, G., Hendren, R., Max, J.E., Paulus, M.P., Tapert, S.F., Banerjee, D., Simmons, A.N., Yang, T.T., 2013. Resting-state functional connectivity of subgenual anterior cingulate cortex in depressed adolescents. Biol. Psychiatry. https://doi.org/10.1016/j.biopsych.2013.05.036

De Kwaasteniet, B., Ruhe, E., Caan, M., Rive, M., Olabarriaga, S., Groefsema, M., Heesink, L., Van Wingen, G., Denys, D., 2013. Relation between structural and functional connectivity in major depressive disorder. Biol. Psychiatry. https://doi.org/10.1016/j.biopsych.2012.12.024

DeRubeis, R.J., Siegle, G.J., Hollon, S.D., 2008. Cognitive therapy versus medication for depression: Treatment outcomes and neural mechanisms. Nat. Rev. Neurosci. https://doi.org/10.1038/nrn2345

Goldapple, K., Segal, Z., Garson, C., Lau, M., Bieling, P., Kennedy, S., Mayberg, H., 2004. Modulation of Cortical-Limbic Pathways in Major Depression: Treatment-Specific Effects of Cognitive Behavior Therapy. Arch. Gen. Psychiatry. https://doi.org/10.1001/archpsyc.61.1.34

Goodyer, I.M., Reynolds, S., Barrett, B., Byford, S., Dubicka, B., Hill, J., Holland, F., Kelvin, R., Midgley, N., Roberts, C., Senior, R., Target, M., Widmer, B., Wilkinson, P., Fonagy, P., 2017. Cognitive behavioural therapy and short-term psychoanalytical psychotherapy versus a brief psychosocial intervention in adolescents with unipolar major depressive disorder (IMPACT): a multicentre, pragmatic, observer-blind, randomised controlled superiori. The Lancet Psychiatry. https://doi.org/10.1016/\$2215-0366(16)30378-9

Grieve, S.M., Korgaonkar, M.S., Koslow, S.H., Gordon, E., Williams, L.M., 2013. Widespread reductions in gray matter volume in depression. Neurolmage Clin. https://doi.org/10.1016/j.nicl.2013.08.016

Hagan, C.C., Graham, J.M.E., Tait, R., Widmer, B., Van Nieuwenhuizen, A.O., Ooi, C., Whitaker, K.J., 
Simas, T., Bullmore, E.T., Lennox, B.R., Sahakian, B.J., Goodyer, I.M., Suckling, J., 2015.

Adolescents with current major depressive disorder show dissimilar patterns of age-related differences in ACC and thalamus. Neurolmage Clin. 7, 391-399.

https://doi.org/10.1016/j.nicl.2014.12.019

Hagan, C.C., Graham, J.M.E., Widmer, B., Holt, R.J., Ooi, C., van Nieuwenhuizen, A.O., Fonagy, P., Reynolds, S., Target, M., Kelvin, R., Wilkinson, P.O., Bullmore, E.T., Lennox, B.R., Sahakian, B.J., Goodyer, I., Suckling, J., 2013. Magnetic resonance imaging of a randomized controlled trial investigating predictors of recovery following psychological treatment in adolescents with moderate to severe unipolar depression: Study protocol for Magnetic Resonance-Improving Mood with Psycho. BMC Psychiatry. https://doi.org/10.1186/1471-244X-13-247

Ho, T.C., Connolly, C.G., Henje Blom, E., LeWinn, K.Z., Strigo, I.A., Paulus, M.P., Frank, G., Max, J.E., Wu, J., Chan, M., Tapert, S.F., Simmons, A.N., Yang, T.T., 2015. Emotion-dependent functional connectivity of the default mode network in adolescent depression. Biol. Psychiatry. https://doi.org/10.1016/j.biopsych.2014.09.002

Hochberg, B., 1995. Controlling the False Discovery Rate: a Practical and Powerful Approach to Multiple Testing. J. R. Stat. Soc. https://doi.org/10.2307/2346101

Holt, R.J., Graham, J.M., Whitaker, K.J., Hagan, C.C., Ooi, C., Wilkinson, P.O., Van Nieuwenhuizen, A.O., Lennox, B.R., Sahakian, B.J., Goodyer, I.M., Bullmore, E.T., Suckling, J., 2016. Functional MRI of emotional memory in adolescent depression. Dev. Cogn. Neurosci. https://doi.org/10.1016/j.den.2015.12.013

Jacobs, R.H., Watkins, E.R., Peters, A.T., Feldhaus, C.G., Barba, A., Carbray, J., Langenecker, S.A., 2016. Targeting ruminative thinking in adolescents at risk for depressive relapse: Ruminationfocused cognitive behavior therapy in a pilot randomized controlled trial with resting state fMRI. PLoS One. https://doi.org/10.1371/journal.pone.0163952

Jenkinson, M., Beckmann, C.F., Behrens, T.E.J., Woolrich, M.W., Smith, S.M., 2012. FSL. Neuroimage. https://doi.org/10.1016/j.neuroimage.2011.09.015

Kaiser, R.H., Andrews-Hanna, J.R., Wager, T.D., Pizzagalli, D.A., 2015. Large-scale network dysfunction in major depressive disorder: A meta-analysis of resting-state functional connectivity. JAMA Psychiatry. https://doi.org/10.1001/jamapsychiatry.2015.0071

Ma, C., Ding, J., Li, J., Guo, W., Long, Z., Liu, F., Gao, Q., Zeng, L., Zhao, J., Chen, H., 2012. RestingState Functional Connectivity Bias of Middle Temporal Gyrus and Caudate with Altered Gray Matter Volume in Major Depression. PLoS One. https://doi.org/10.1371/journal.pone.0045263

Manoliu, A., Meng, C., Brandl, F., Doll, A., Tahmasian, M., Scherr, M., Schwerthöffer, D., Zimmer, C., Förstl, H., Bäuml, J., Riedl, V., Wohlschläger, A.M., Sorg, C., 2014. Insular dysfunction within the salience network is associated with severity of symptoms and aberrant inter-network connectivity in major depressive disorder. Front. Hum. Neurosci. https://doi.org/10.3389/fnhum.2013.00930

Mayberg, H.S., 1997. Limb ic-Cortical Dysregulation: A Proposed Model of Depression. J. Neuropsychiatry Clin. Neurosci. https://doi.org/10.1176/jnp.9.3.471

Mulders, P.C., van Eijndhoven, P.F., Schene, A.H., Beckmann, C.F., Tendolkar, I., 2015. Resting-state functional connectivity in major depressive disorder: A review. Neurosci. Biobehav. Rev. https://doi.org/10.1016/j.neubiorev.2015.07.014

Pannekoek, J.N., Van Der Werff, S.J.A., Meens, P.H.F., Van Den Bulk, B.G., Jolles, D.D., Veer, I.M., Van Lang, N.D.J., Rombouts, S.A.R.B., Van Der Wee, N.J.A., Vermeiren, R.R.J.M., 2014. Aberrant 
resting-state functional connectivity in limbic and salience networks in treatment-naïve clinically depressed adolescents. J. Child Psychol. Psychiatry Allied Discip.

https://doi.org/10.1111/jcpp.12266

Peterson, B.S., Warner, V., Bansal, R., Zhu, H., Hao, X., Liu, J., Durkin, K., Adams, P.B., Wickramaratne, P., Weissman, M.M., 2009. Cortical thinning in persons at increased familial risk for major depression. Proc. Natl. Acad. Sci. U. S. A. https://doi.org/10.1073/pnas.0805311106

Ramasubbu, R., Konduru, N., Cortese, F., Bray, S., Gaxiola-Valdez, I., Goodyear, B., 2014. Reduced intrinsic connectivity of amygdala in adults with major depressive disorder. Front. Psychiatry. https://doi.org/10.3389/fpsyt.2014.00017

Reynolds, S., Carrey, N., Jaworska, N., Langevin, L.M., Yang, X.-R., MacMaster, F.P., 2014. Cortical thickness in youth with major depressive disorder. BMC Psychiatry. https://doi.org/10.1186/1471-244X-14-83

Ritchey, M., Dolcos, F., Eddington, K.M., Strauman, T.J., Cabeza, R., 2011. Neural correlates of emotional processing in depression: Changes with cognitive behavioral therapy and predictors of treatment response. J. Psychiatr. Res. https://doi.org/10.1016/j.jpsychires.2010.09.007

Roiser, J.P., Sahakian, B.J., 2013. Hot and cold cognition in depression. CNS Spectr. https://doi.org/10.1017/S1092852913000072

Schmaal, L., Hibar, D.P., Sämann, P.G., Hall, G.B., Baune, B.T., Jahanshad, N., Cheung, J.W., Van Erp, T.G.M., Bos, D., Ikram, M.A., Vernooij, M.W., Niessen, W.J., Tiemeier, H., Hofman, A., Wittfeld, K., Grabe, H.J., Janowitz, D., Bülow, R., Selonke, M., Völzke, H., Grotegerd, D., Dannlowski, U., Arolt, V., Opel, N., Heindel, W., Kugel, H., Hoehn, D., Czisch, M., Couvy-Duchesne, B., Rentería, M.E., Strike, L.T., Wright, M.J., Mills, N.T., De Zubicaray, G.I., McMahon, K.L., Medland, S.E., Martin, N.G., Gillespie, N.A., Goya-Maldonado, R., Gruber, O., Krämer, B., Hatton, S.N., Lagopoulos, J., Hickie, I.B., Frodl, T., Carballedo, A., Frey, E.M., Van Velzen, L.S., Penninx, B.W.J.H., Van Tol, M.J., Van der Wee, N.J., Davey, C.G., Harrison, B.J., Mwangi, B., Cao, B., Soares, J.C., Veer, I.M., Walter, H., Schoepf, D., Zurowski, B., Konrad, C., Schramm, E., Normann, C., Schnell, K., Sacchet, M.D., Gotlib, I.H., MacQueen, G.M., Godlewska, B.R., Nickson, T., McIntosh, A.M., Papmeyer, M., Whalley, H.C., Hall, J., Sussmann, J.E., Li, M., Walter, M., Aftanas, L., Brack, I., Bokhan, N.A., Thompson, P.M., Veltman, D.J., 2017. Cortical abnormalities in adults and adolescents with major depression based on brain scans from 20 cohorts worldwide in the ENIGMA Major Depressive Disorder Working Group. Mol. Psychiatry. https://doi.org/10.1038/mp.2016.60

Sharp, C., Goodyer, I.M., Croudace, T.J., 2006. The Short Mood and Feelings Questionnaire (SMFQ): A unidimensional item response theory and categorical data factor analysis of self-report ratings from a community sample of 7-through 11-year-old children. J. Abnorm. Child Psychol. https://doi.org/10.1007/s10802-006-9027-x

Shou, H., Yang, Z., Satterthwaite, T.D., Cook, P.A., Bruce, S.E., Shinohara, R.T., Rosenberg, B., Sheline, Y.I., 2017. Cognitive behavioral therapy increases amygdala connectivity with the cognitive control network in both MDD and PTSD. Neurolmage Clin. https://doi.org/10.1016/j.nicl.2017.01.030

Sowell, E.R., Peterson, B.S., Thompson, P.M., Welcome, S.E., Henkenius, A.L., Toga, A.W., 2003. Mapping cortical change across the human life span. Nat. Neurosci. https://doi.org/10.1038/nn1008

Späti, J., Hänggi, J., Doerig, N., Ernst, J., Sambataro, F., Brakowski, J., Jäncke, L., Grosse Holtforth, M., Seifritz, E., Spinelli, S., 2015. Prefrontal thinning affects functional connectivity and regional homogeneity of the anterior cingulate cortex in depression. Neuropsychopharmacology. 
https://doi.org/10.1038/npp.2015.8

Spielberger, C.D., Gorsuch, R.L., Lushene, R.E., 1970. The State-Trait Anxiety Inventory. MANUAL. https://doi.org/10.1037/t06496-000

Steingard, R.J., Renshaw, P.F., Hennen, J., Lenox, M., Cintron, C.B., Young, A.D., Connor, D.F., Au, T.H., Yurgelun-Todd, D.A., 2002. Smaller frontal lobe white matter volumes in depressed adolescents. Biol. Psychiatry. https://doi.org/10.1016/S0006-3223(02)01393-8

Tzourio-Mazoyer, N., Landeau, B., Papathanassiou, D., Crivello, F., Etard, O., Delcroix, N., Mazoyer, B., Joliot, M., 2002. Automated anatomical labeling of activations in SPM using a macroscopic anatomical parcellation of the MNI MRI single-subject brain. Neuroimage.

https://doi.org/10.1006/nimg.2001.0978

Van Tol, M.J., Li, M., Metzger, C.D., Hailla, N., Horn, D.I., Li, W., Heinze, H.J., Bogerts, B., Steiner, J., He, H., Walter, M., 2014. Local cortical thinning links to resting-state disconnectivity in major depressive disorder. Psychol. Med. https://doi.org/10.1017/S0033291713002742

Wang, L., Hermens, D.F., Hickie, I.B., Lagopoulos, J., 2012. A systematic review of resting-state functional-MRI studies in major depression. J. Affect. Disord.

https://doi.org/10.1016/j.jad.2012.04.013

Weersing, V.R., Jeffreys, M., Do, M.C.T., Schwartz, K.T.G., Bolano, C., 2017. Evidence Base Update of Psychosocial Treatments for Child and Adolescent Depression. J. Clin. Child Adolesc. Psychol. https://doi.org/10.1080/15374416.2016.1220310

Whitaker, K.J., Vértes, P.E., Romero-Garcia, R., Váša, F., Moutoussis, M., Prabhu, G., Weiskopf, N., Callaghan, M.F., Wagstyl, K., Rittman, T., Tait, R., Ooi, C., Suckling, J., Inkster, B., Fonagy, P., Dolan, R.J., Jones, P.B., Goodyer, I.M., Bullmore, E.T., 2016. Adolescence is associated with genomically patterned consolidation of the hubs of the human brain connectome. Proc. Natl. Acad. Sci. 113, 9105-9110. https://doi.org/10.1073/pnas.1601745113

Yoshimura, S., Okamoto, Y., Matsunaga, M., Onoda, K., Okada, G., Kunisato, Y., Yoshino, A., Ueda, K., Suzuki, S. ichi, Yamawaki, S., 2017. Cognitive behavioral therapy changes functional connectivity between medial prefrontal and anterior cingulate cortices. J. Affect. Disord. https://doi.org/10.1016/j.jad.2016.10.017

Zeng, L.-L., Liu, L., Liu, Y., Shen, H., Li, Y., Hu, D., 2012. Antidepressant Treatment Normalizes White Matter Volume in Patients with Major Depression. PLoS One.

https://doi.org/10.1371/journal.pone.0044248 


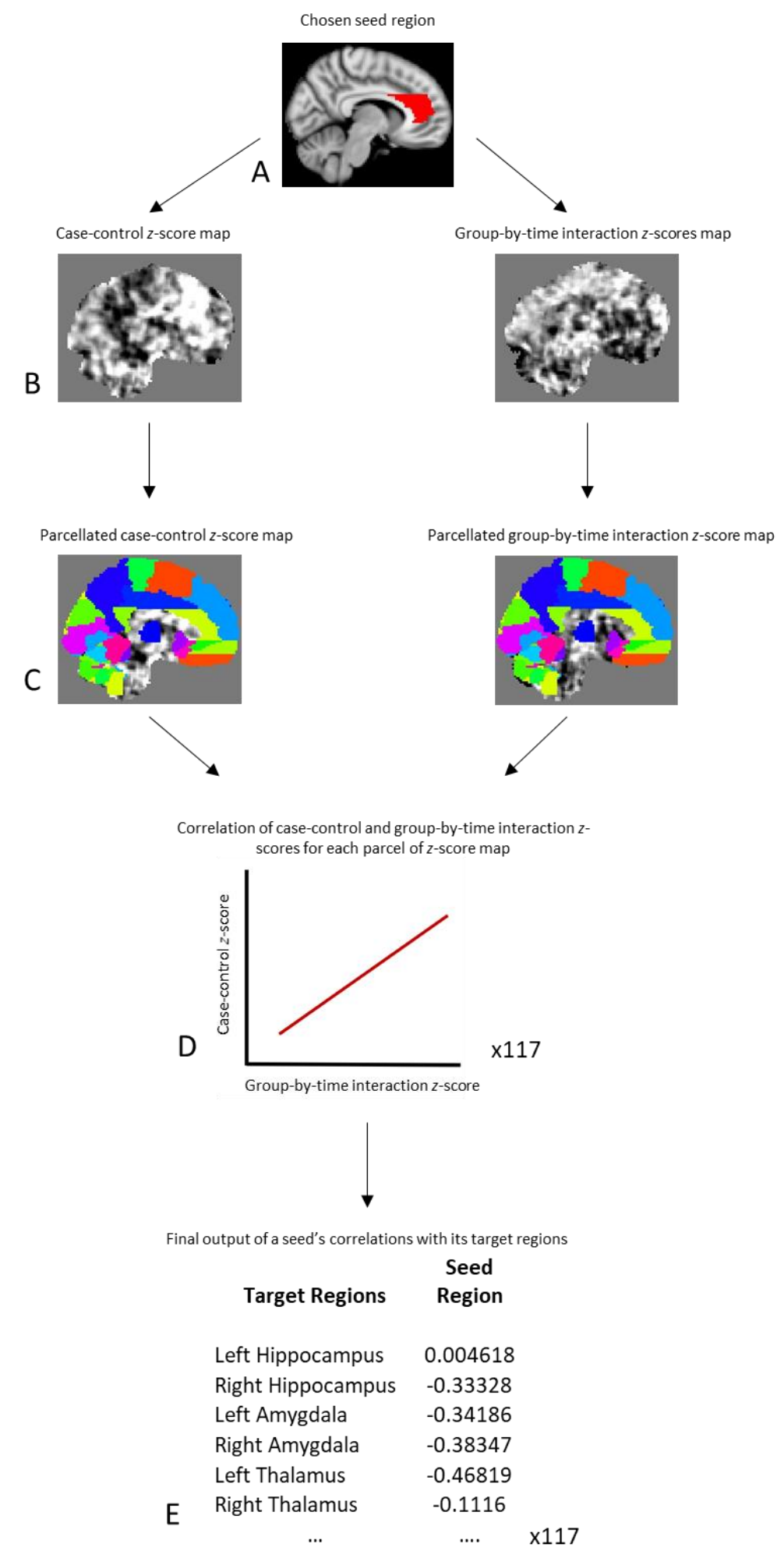

Figure 1. Demonstrates the method used to investigate the relationship between pre-treatment functional disruption and cognitive behavioral therapy-related changes in resting-state functional connectivity. A) Shows an example of a seed region used. B) Shows the case-control and group-by-time interaction z-score maps generated by the seed analysis. C) Shows both of the $z$-score maps being parcellated into the 117 target regions. D) Demonstrates a correlation between the case-control and group-by-time $z$-scores of a certain target region. E) Shows the final results of a seed regions correlations corresponding to each target region. 

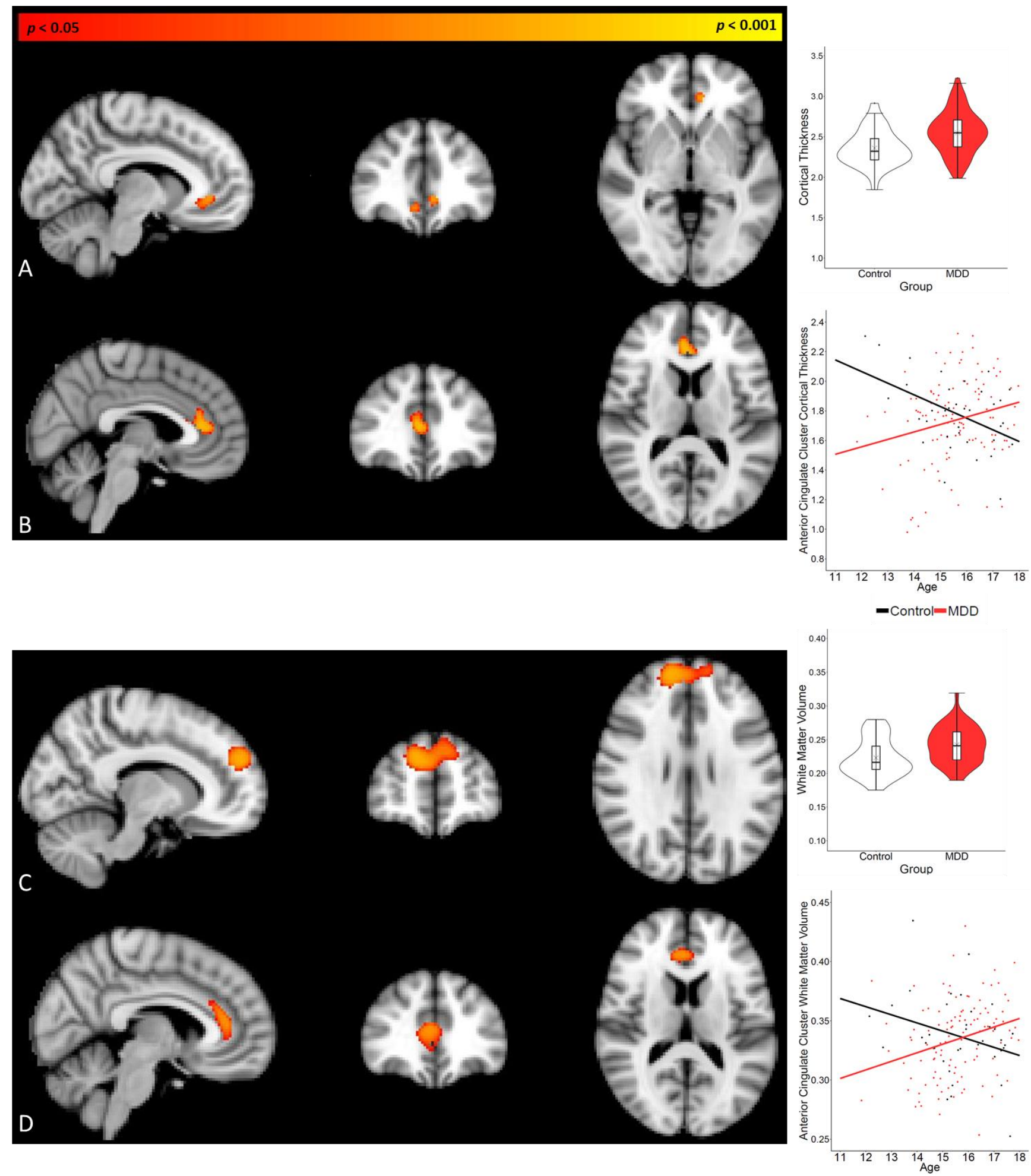

Figure 2 Shows the case-control differences in cortical thickness and white matter volume, between adolescent MDD patients and controls within the cross-sectional sample. A) Patients showed greater cortical thickness within the bilateral subgenual anterior cingulate and medial orbitofrontal cortex. B) Shows the significant group-by-age interaction in cortical thickness, within the pregenual anterior cingulate. C) Shows the significant cluster where patients showed greater white matter volume within the bilateral medial frontal gyri, bilateral superior frontal gyri and right middle frontal gyrus. D) Shows the significant group-by-age interaction in white matter volume, within the bilateral pregenual anterior cingulate and right subgenual anterior cingulate. 


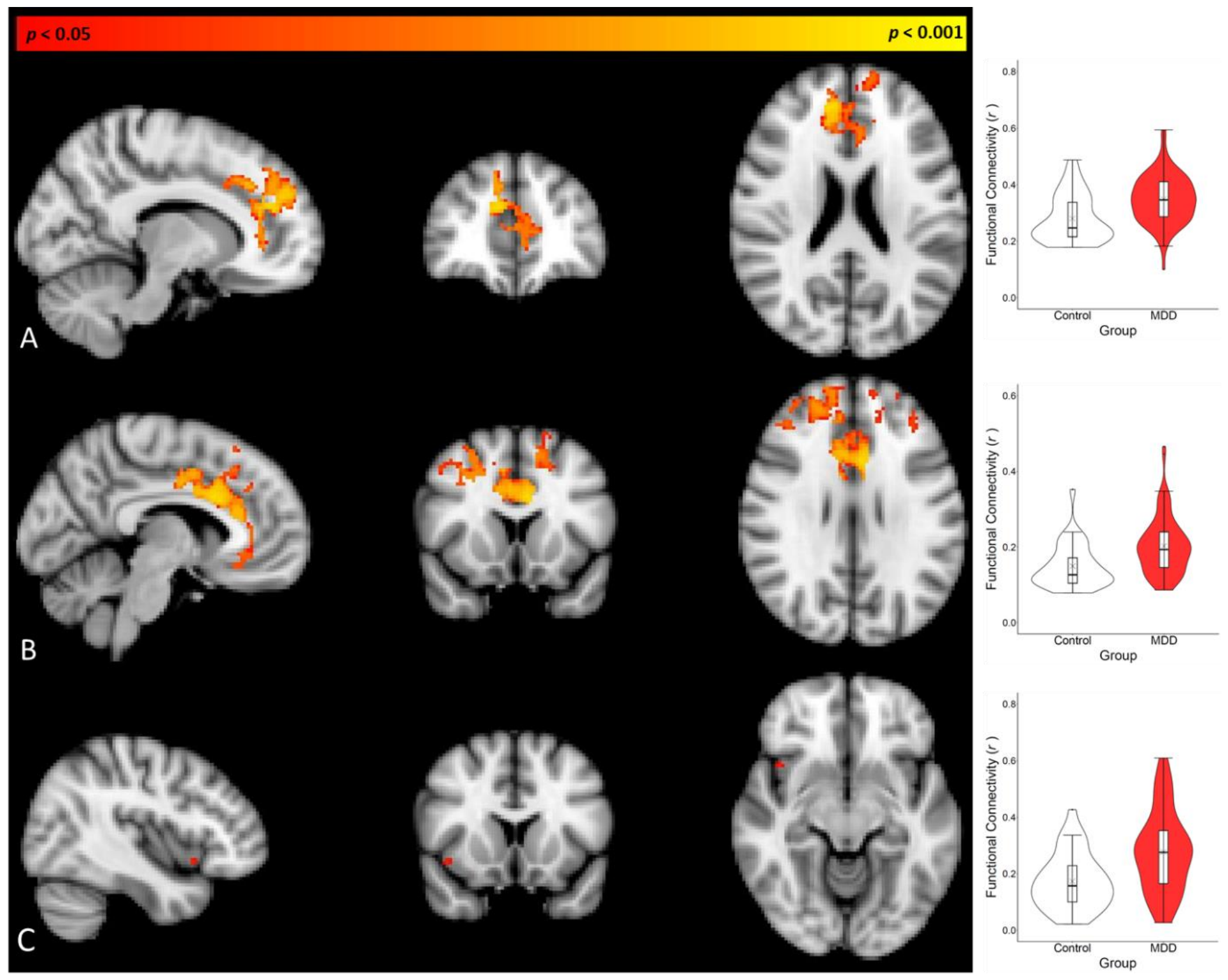

Figure 3. Shows Clusters and violin plots of greater resting state functional connectivity in patients in the cross-sectional sample. A: Right Superior Frontal Gyrus Seed, B: Right Subgenual Anterior Cingulate Seed, C: Right Amygdala Seed. 

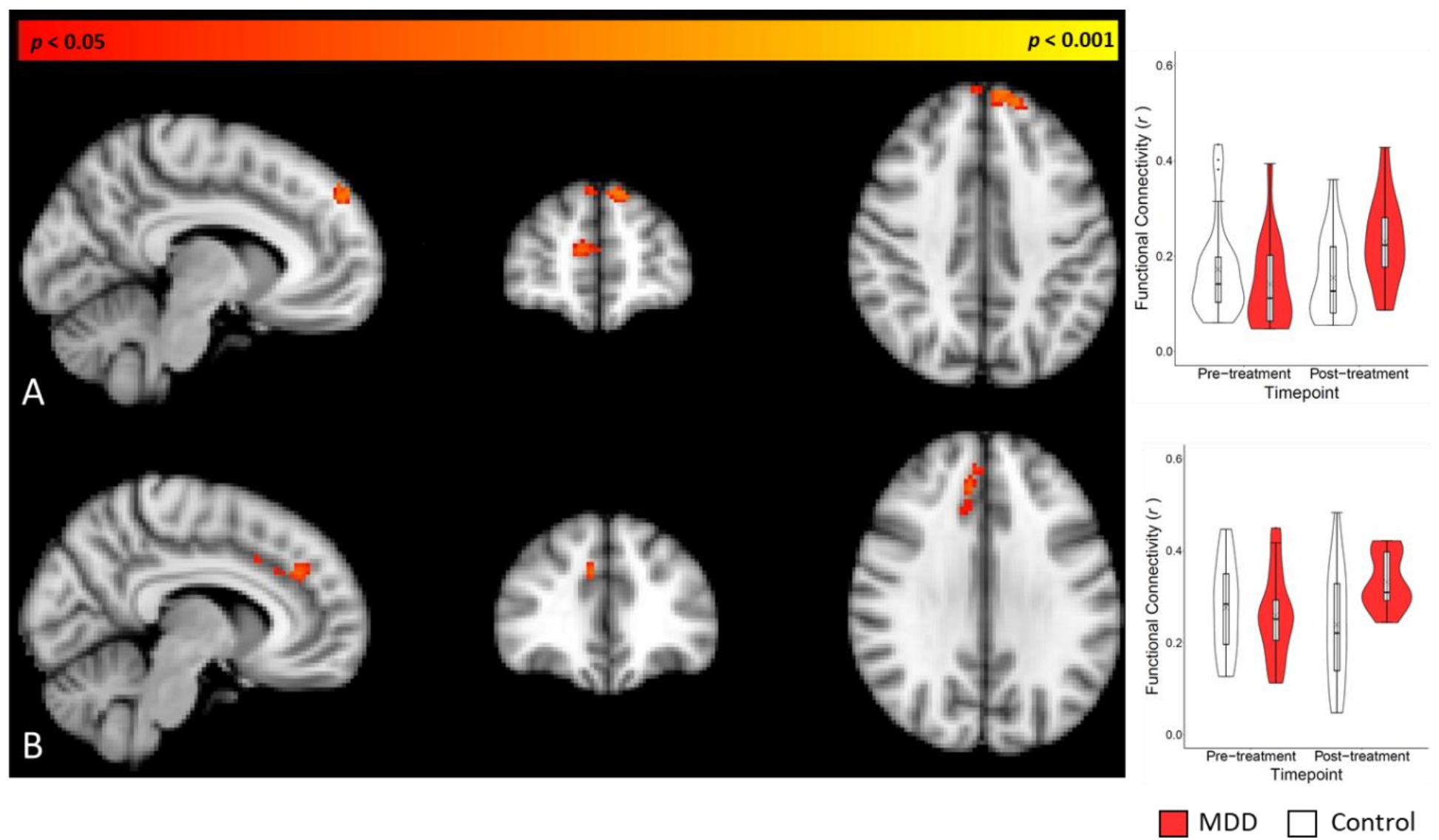

Figure 4. Shows the group-by-time interactions in resting-state functional connectivity, and their violin plots, in the longitudinal sample. A: Left Amygdala Seed, B: Left Subgenual Anterior Cingulate Cortex Seed. 
Averaged $r^{2}$ for positive correlations

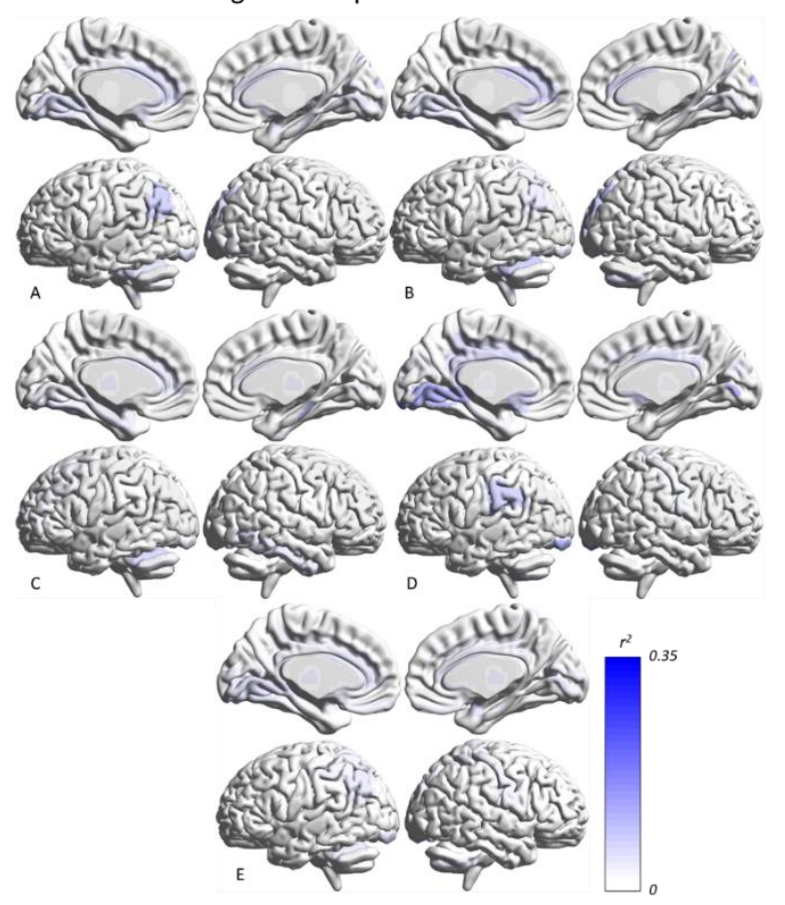

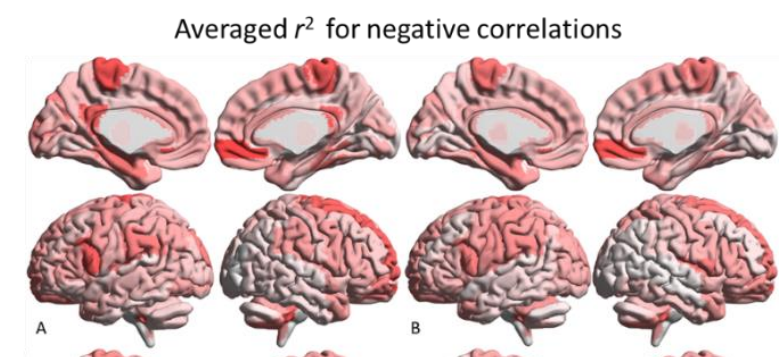
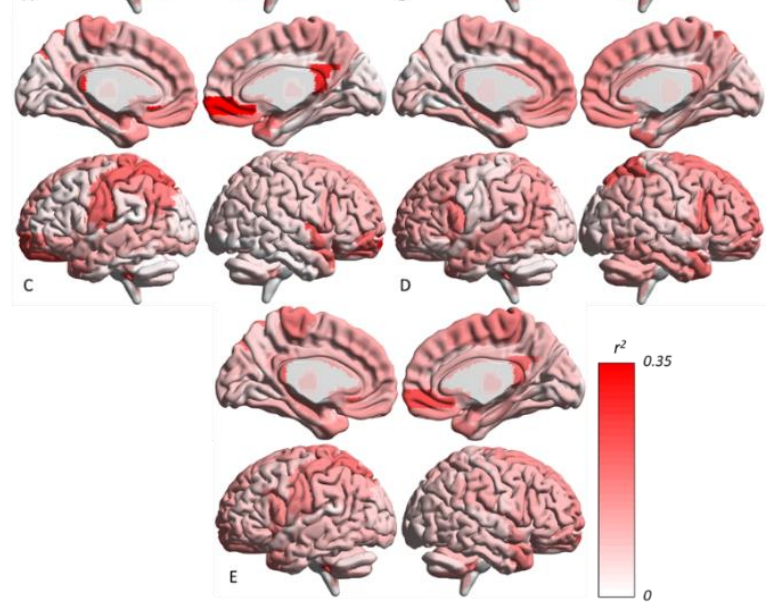

Top $10 \%$ strongest negative correlations
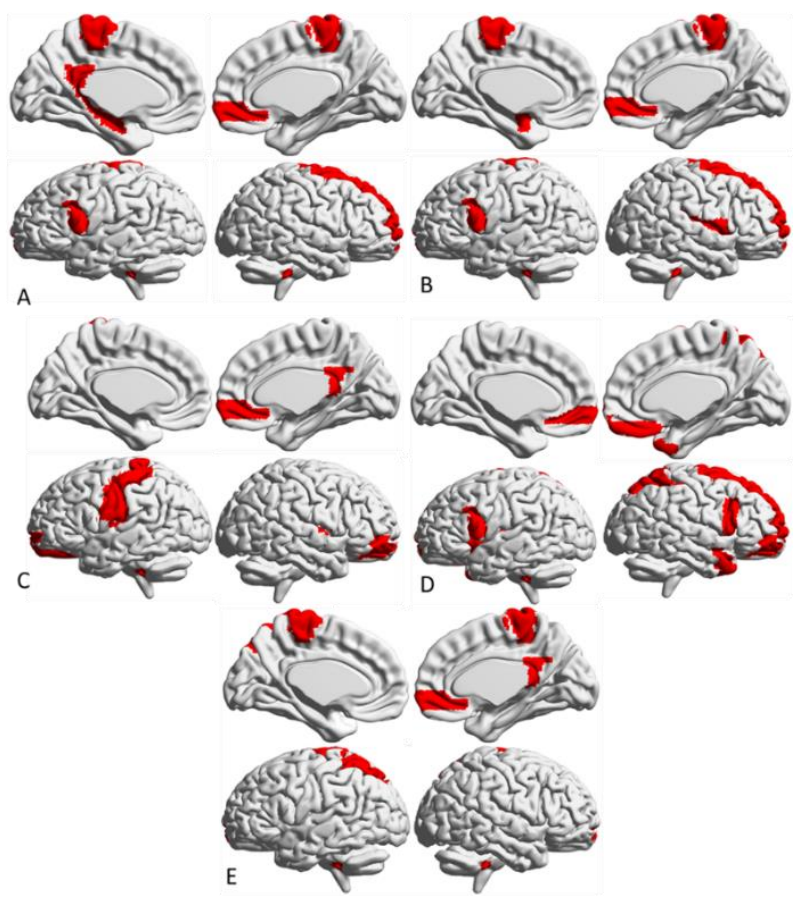

Figure 5. Shows the averaged $r^{2}$ values, across each seed region grouping, for positive correlations between pre-treatment case-control z-scores and group-by-time interaction z-scores (left), and negative correlations between pre-treatment casecontrol z-scores and group-by-time interaction z-scores (right). It also shows the top $10 \%$ strongest negative correlations, between pre-treatment case-control z-scores and group-by-time interaction z-scores, for each seed region grouping (bottom). A: Fronto-limbic network. B: Default mode network. C: Central executive network. D: Salience network. E: Average across all 118 seed regions. 